\title{
Barriers to ideal outcomes after pediatric liver transplantation
}

\author{
Vicky Lee $\mathrm{Ng}^{1}$ (D) | George V. Mazariegos ${ }^{2}$ (D) | Beau Kelly ${ }^{3}$ | Simon Horslen ${ }^{4}$ (D) | \\ Sue V. McDiarmid ${ }^{5}$ | John C. Magee ${ }^{6}$ | Kathleen M. Loomes ${ }^{7}$ | Ryan T. Fischer ${ }^{8}$ | \\ Shikha S. Sundaram ${ }^{9}$ | Jennifer C. Lai ${ }^{10} \mid$ Helen S. Te ${ }^{11}$ John C. Bucuvalas ${ }^{12}$ \\ ${ }^{1}$ Division of Pediatric Gastroenterology, Hepatology and Nutrition, Transplant and Regenerative Medicine Center, The Hospital for Sick Children, \\ University of Toronto, Toronto, Ontario, Canada \\ ${ }^{2}$ Hillman Center for Pediatric Transplantation, Children's Hospital of Pittsburgh of UPMC, Pittsburgh, Pennsylvania \\ ${ }^{3}$ Division of Surgery, DCI Donor Services, Sacramento, California \\ ${ }^{4}$ Department of Pediatrics, University of Washington, Seattle, Washington \\ ${ }^{5}$ David Geffen School of Medicine, University of California, Los Angeles, California, USA \\ ${ }^{6}$ Department of Surgery, University of Michigan Medical School, Ann Arbor, Michigan \\ ${ }^{7}$ Division of Gastroenterology, Hepatology and Nutrition, Children's Hospital of Philadelphia, University of Pennsylvania Perelman School of Medicine, \\ Philadelphia, Pennsylvania \\ ${ }^{8}$ Division of Gastroenterology, Hepatology and Nutrition, Children's Mercy Hospital, University of Missouri-Kansas City School of Medicine, Kansas City, \\ Missouri \\ ${ }^{9}$ Pediatrics, Gastroenterology, Hepatology and Nutrition, Children's Hospital Colorado, University of Colorado School of Medicine, Aurora, Colorado \\ ${ }^{10}$ Division of Gastroenterology/Hepatology, Department of Medicine, University of California, San Francisco, San Francisco, California \\ ${ }^{11}$ Adult Liver Transplant Program, University of Chicago Medicine, Chicago, Illinois \\ ${ }^{12}$ Mount Sinai Kravis Childrens Hospital and Recanati/Miller Transplant Institute, New York City, New York
}

\section{Correspondence}

Vicky Lee Ng, Division of Pediatric Gastroenterology, Hepatology and Nutrition, Transplant and Regenerative Medicine Center, The Hospital for Sick Children, University of Toronto, Toronto, ON, Canada.

Email: vicky.ng@sickkids.ca

\section{Funding information}

This study was funded in part by $\mathrm{NIH}$ K23AG048337 (Lai), NIH R01AG059183 (Lai). These funding agencies played no role in the analysis of the data or the preparation of this manuscript. Its contents are solely the responsibility of the authors and do not necessarily represent the official views of the $\mathrm{NIH}$.

\begin{abstract}
Long-term survival for children who undergo LT is now the rule rather than the exception. However, a focus on the outcome of patient or graft survival rates alone provides an incomplete and limited view of life for patients who undergo LT as an infant, child, or teen. The paradigm has now appropriately shifted to opportunities focused on our overarching goals of "surviving and thriving" with long-term allograft health, freedom of complications from long-term immunosuppression, self-reported wellbeing, and global functional health. Experts within the liver transplant community highlight clinical gaps and potential barriers at each of the pretransplant, intra-operative, early-, medium-, and long-term post-transplant stages toward these broader mandates. Strategies including clinical research, innovation, and quality improvement targeting both traditional as well as PRO are outlined and, if successfully leveraged and conducted, would improve outcomes for recipients of pediatric LT.
\end{abstract}

Abbreviations: AASLD, American Association for the Study of Liver Diseases; AST, American Society of Transplantation; DDLT, deceased donor liver transplantation; FTR, failure to rescue; HRQOL, Health related Quality of Life; LDLT, living donor liver transplantation; LICOP, liver and intestinal community of practice; LT, liver transplantation; MLVI, Medication Level Variability Index; NASPGHAN, North American Society for Pediatric Gastroenterology, Hepatology, and Nutrition; NSER, non-standard exception requests; PCOP, pediatric community of practice; PELD, pediatric end-stage liver disease; PeLTQL, pediatric liver transplant quality of life; PICU, pediatric intensive care unit; PRO, patient-reported outcomes; PROM, Patient Reported Outcome Measure; PTLD, post-transplant lymphoproliferative disease; SNEPT, Starzl Network for Excellence in Pediatric Transplantation; SPLIT, Society of Pediatric Liver Transplant; SRTR, Scientific Registry of Transplant Recipients. 


\section{1 | INTRODUCTION}

The first LT was performed by Thomas Starzl in 1963 on a 2-year-old child with biliary atresia, proving that hepatic replacement surgery was technically feasible. ${ }^{1}$ Effective immunosuppression, improved technical expertise enabling technical variant grafts and LDLT, ${ }^{2}$ and expanded therapies to prevent infection are a few examples of key contributors to improved 5-year patient and graft survival rates upwards of $90 \% .^{3}$ However, survival metrics provide an incomplete and limited view of life after LT in infants, children, teens, and young adults. The emerging paradigm is now appropriately shifted to opportunities focused on enhancing the health span, defined as the length of time that a pediatric recipient is alive and functionally well following transplantation. At 10 years after LT, less than $1 / 3$ of pediatric recipients registered in the SPLIT registry are considered to be in "ideal or optimal health," as defined by (a) normal liver tests; (b) maintenence immunosuppression monotherapy; (c) normal growth; and (d) free of significant immunosuppression-associated comorbid conditions. ${ }^{4}$ Of note, this ideal outcome estimate is almost certainly optimistic given emerging findings highlighting that consistently normal results of liver tests "do not tell the whole story" and may in fact hide a spectrum of subclinical pathology that can only be accurately revealed by histopathological examination of tissue provided by a liver biopsy. ${ }^{5-7}$ The goals of pediatric LT mandate targeted strategies to ensure children thrive as well as survive. However, pediatric
LT management and care is challenging and highly specialized, with psychosocial development during the adolescent years adding particular complexities. Research is limited by the power of study size, logistical impediments of being integrated within larger or complex hospital systems, and eventual transfer of care to adult transplant centers. Subsequent barriers to collaboration include geospatial location, current metrics of clinical productivity, matrix reporting structures, increasing regulatory requirements and demands for professional advancement. ${ }^{8}$

A group of experts in the LT community identified and summarized key barriers which, if successfully leveraged and conducted, would optimize the unpredictable and often circuitous journey for young children with irreversible liver disease for whom LT offers the only chance for long-term survival. This manuscript proposes constructs to guide a path forward in addressing these barriers and advancing pediatric LT care.

\subsection{Journey of the pediatric patient}

Each child who is evaluated and accepted as a candidate for LT embarks on a unique journey with potential obstacles and hurdles which may challenge attainment of the end goal of sustainable long-term health (Figure 1). This journey to best outcomes must traverse a broad landscape, with multiple phases beginning with listing decisions, addressing predictable challenges pretransplant,

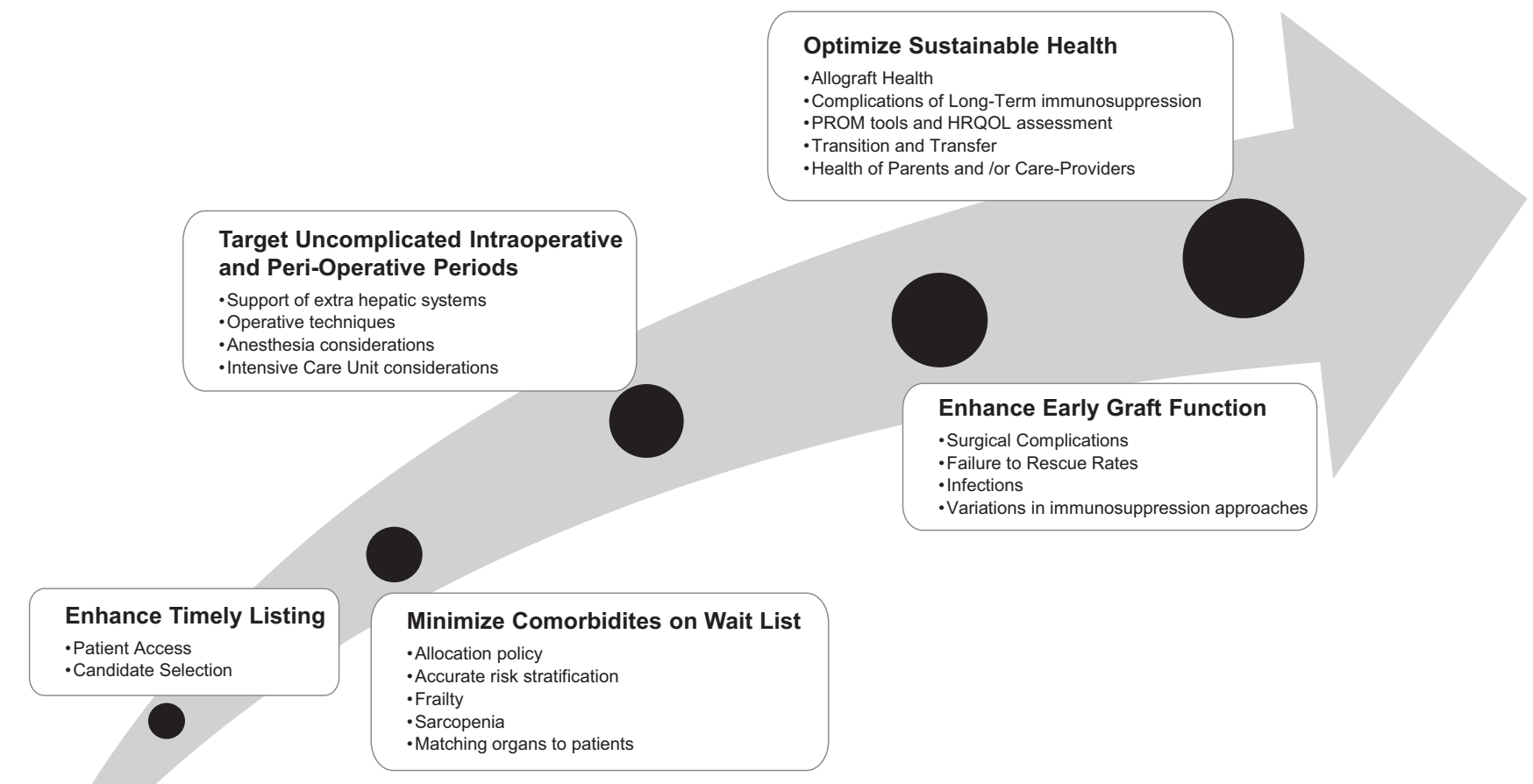

FIG URE 1 Journey of the patient following pediatric Liver Transplantation - Key Factors Contributory to Desired Outcomes. HRQOL, Health related Quality of Life; PROM, Patient Reported Outcome Measure 
and the recovery process with continuous learning and adaptation to care during the post-transplant course. The combinations of challenges are unique for each patient. Timely listing requires early recognition of $\mathrm{LT}$ responsive disease conditions, easy access to LT care, and fulfillment of LT center listing criteria. Once listed, the goals shift to surviving the wait list without incurring significant morbidity until a suitable donor organ is available. Variations in allocation policies and physician organ acceptance behavior and practices exist among countries and between LT centers. ${ }^{9}$ Once the "right" donor liver is identified, the patient must undergo the LT operation and peri-operative phases, prior to navigating the early-, medium-, and long-term post-transplant follow-up care phases. Multiple elements of each phase of care will inform graft function, which are instrumental and contributory to optimizing both life span and health span for pediatric LT recipients. The best patientcentered outcomes reflect a complex care cycle in which each intervention is dependent on the effectiveness of previous decisions and interventions, with active participation toward optimizing the child's overall health before and after LT.

\section{2 | Timely listing for all pediatric LT candidates}

\subsubsection{Access to care and candidate selection}

Despite formal guidelines for the evaluation and selection of the pediatric LT patient endorsed by the AASLD, AST, and NASPGHAN, ${ }^{10}$ empirical evidence suggests that there is substantial variation among listing decision-making among centers. ${ }^{11}$ There are more than 30 pediatric liver transplant centers in the United States and three pediatric programs in Canada that performed five or more pediatric LT in the calendar year 2018 (https://optn.transplant.hrsa.gov/ data/view-data-reports/build-advanced, accessed March 17, 2019). The travel distance from the patient's home to the LT center varies greatly. Potential contributors limiting access include insurance type, and racial and gender disparities. ${ }^{11}$

The more common indications for LT including biliary atresia, autoimmune liver disease, and genetic disorders of intrahepatic cholestasis for LT are generally readily identified by referring physicians although delayed diagnoses continue to challenge. There is a lack of objective metrics to assess non-hepatic morbidity for inherited defect of metabolism, especially in those conditions not associated with structural liver disease. Whether LT is indicated for inherited disorders of metabolism, cystic fibrosis, liver fibrosis associated with congenital heart disease and complex hepatic tumors, among others, may be challenging to determine since decision-making requires coordination of care across disciplines. ${ }^{12-14}$ Delays are particularly critical since insults during the early years impact brain development, growth, and long-term functional health, as well as vulnerabilities specific to pediatrics. ${ }^{15}$ The expanding indications of pediatric liver diseases amenable to $\mathrm{LT}$, the relatively small number of pediatric patients, and the challenges of extrapolating adult data to children challenge prognostication. Indeed, the expected 90-day mortality using the PELD underestimated the actual probability of death by as much as $17 \% .{ }^{16}$ Additional considerations impacting candidate selection include individual center medical and surgical experience, clinical decision-making, resource allocation, execution of the operational standards, and impact of center-specific outcomes reported to the public domain. ${ }^{17}$

Recommendations

- Characterize factors contributing to delays in referral of pediatric patient to LT centers.

- Develop strategies to mitigate the barriers to timely access to LT centers for pediatric patients with indications for LT.

- Develop a strategy to track outcomes for those who are not selected as LT candidates.

- Standardize selection criteria within and among pediatric centers.

\subsection{2 | Decreasing time duration and minimizing comorbidities on the wait list}

The impact of wait time duration and the ensuing comorbidities are not equivalent between developing children and adults. Children with irreversible liver disease offer a more complex challenge since crucial and time-limited opportunities for growth, cognitive, neurological, and social development are lost with increasing wait list duration leading to more procedures and hospitalizations for medical therapies and need for prehabilitation optimization strategies.

\subsection{3 | Allocation}

In the United States, the PELD score is used to prioritize children on the wait list. Since its implementation, additional points for children are available either by automatic granting of "standard exception points" for a list of consensus conditions, or by petitioning for individual case-by-case "NSER" by pediatric LT program submitting narratives explaining why a calculated PELD score does not accurately reflect mortality or morbidity risk for a specific patient. Currently, there is lack of standardization in NSER utilization overall. However, NSER denial increases the risk of wait list mortality or removal from the wait list for being too sick. ${ }^{18}$ Despite $5 \%-10 \%$ yearly increases in organ donation and concurrent increments in total transplants performed annually, death or "removal for too sick to transplant" rates range from $7 \%$ to $12 \%$ on the pediatric LT wait list, ${ }^{19,20}$ while the number of pediatric LTs performed yearly has remained relatively fixed. The feasibility of a decrease in pediatric wait list deaths, concurrent with an overall increase in LTs performed, was recently demonstrated in a liver simulated allocation model analysis prioritizing pediatric plus the sickest (Status 1 ) adult patients for deceased donor livers utilizing data from the SRTR. ${ }^{21}$

Recommendations

- Optimize organ allocation policies and develop risk stratification systems to improve access and reduce wait list mortality among children. 


\subsection{4 | Distribution}

The disparity of requests and approval for exception is sobering, with patients of white race and private insurance more likely to benefit. Receiving an exception translated into a nearly 3 -fold increased likelihood of transplantation. ${ }^{22}$ Organ acceptance rates and utilization of technical variant organs from deceased donors also vary among pediatric LT centers, which may further impact the risk for children. Racial disparities in access to LT have been attributed to multi-factorial causes including biologic, socio-economic, and cultural factors. ${ }^{11}$ There is disparity in use of exceptions by race that is not explained by clinical disease severity, primary diagnosis, geography, or other demographic factors. ${ }^{22}$ Understanding the presence and ultimately the root causes of these disparities in pediatric LT will enhance opportunities to save the lives of more children on the wait list. DDLT remains the standard of care for LT in North American centers. The demand for feasible DD livers far exceeds the available supply, and opportunities for LDLT to be used more widely by pediatric LT programs are warranted to reduce or eliminate wait list deaths, improve time to transplant, and ultimately improve long-term outcomes for children in need of LT. ${ }^{2}$

\section{Recommendations}

- Develop methodologies targeting disparities in timely access to quality organs in pediatric transplantation.

- Track outcomes of live donor LT by pediatric transplant programs, including wait list deaths, time to transplant, and comorbidities from shortened time to transplant surgery, including LT with livers from anonymous donors.

\section{3 | Enhancing risk stratification of patients on the wait list}

The "state of health" of children with end-stage liver disease on the wait list is influenced not only by the severity of their liver disease, but also by factors such as nutritional status, functional impairments, and non-liver related comorbidities including renal failure or cardiopulmonary disease. These latter factors contribute to wait list outcomes, yet are not well captured by the PELD score. Recent review of two-dimensional echocardiographic findings in listed children with biliary atresia demonstrates that cirrhotic cardiomyopathy is independently associated with serious adverse events and peritransplant death. ${ }^{23}$ The biologic construction of physical frailty, originally developed in the field of geriatrics, has been shown to capture the effects of end-stage liver failure in adults ("relative chronologic youth") and predict post-transplant outcomes and morbidity. ${ }^{24-26}$ The concept of frailty has been extended to children, with a recent study demonstrating feasibility of testing for the five domains of the Fried Frailty Phenotype in a multicenter cohort of children between the ages of 5 and 18 years with chronic liver disease, with $46 \%$ meeting the criteria for frailty at a single baseline assessment. ${ }^{27}$ However, the majority of wait listed children are under the age of 5 years, and thereby too young to undergo functional or performance-based (frailty) testing before time of LT. Pilot sarcopenia studies have demonstrated that children with end-stage liver disease have smaller psoas muscle areas on CT abdominal imaging than healthy agematched controls, and that the psoas muscle area does not correlate with weight z-scores nor PELD score. ${ }^{28,29}$

\section{Recommendations}

- Develop validated and objective pediatric tools and non-invasive biomarkers to more fully capture global functional health, nutritional status, and neurocognitive development.

- Develop more accurate metrics that portend risk beyond those provided by measures of liver injury and function, prioritizing high yield areas of frailty, sarcopenia, and non-invasive biomarkers which may aid risk stratification of children and adolescents on the liver transplant wait list.

\subsection{Minimizing risks during the intra- and peri- operative phases}

Minimizing complications in the intra-operative and early post-operative periods requires effective decision-making in patient selection and the organ to be used. Critical components include preoperative medical evaluation and optimization of the recipient's health prior to LT (including avoiding delays of childhood immunizations), simultaneous coordination and execution of the donor and recipient operations, and the effective medical management of the patient in the operating room and post-operatively in the PICU. In aviation safety research, many if not all fatal crashes were root-caused to a preventable complication of poor communication, execution, or problem recognition, ${ }^{30,31}$ concepts that extend to peri-operative graft loss or patient mortality. ${ }^{32}$ For instance, both duration of extubation and length of PICU stay are actionable determinants that could decrease overall length of stay, mitigate risk, and minimize wastage of valuable resources. ${ }^{33,34}$ In order to optimize outcomes following LT, clinicians must adopt approaches to not only prevent complications, but also to successfully salvage patients after complications occur. ${ }^{35}$ While some complications may not be preventable, the ability to rapidly diagnose and "rescue" a patient from a complication (minimizing the rates of FTR) relates to the quality of care of the health by the healthcare system. This strategy can help identify important areas for outcome improvements across centers and is emerging as a useful quality improvement tool for the pediatric LT field. ${ }^{36}$

\section{Recommendations}

- Develop checklists of intra-operative events and their timing that could predict the likelihood that a patient will progress as expected in their post-operative care versus requiring subsequent planned or unplanned operations that may prolong recovery.

- Identify center practice variations in peri-operative and PICU care and minimize failure-to-rescue rates. 


\section{5 | Long-term health after LT}

\subsection{1 | Allograft health}

Meticulous management of immunosuppression is required to ensure the best long-term outcomes for all LT recipients. In pediatrics, this theme is uniquely challenging given the goal of multiple decades of graft and patient survival. "Too little" immunosuppression risks chronic and often subclinical alloimmune injury, while "too much" immunosuppression risks insidious and cumulative toxicities and comorbidities affecting extra-hepatic organ systems. Immunosuppression after pediatric LT is dominantly driven by program preference, although patient or donor characteristics clearly affect initial regimen choice. ${ }^{37}$ Children, with their longer life expectancy, experience greater cumulative exposure to immunosuppressive agents, thereby increasing their potential morbidity from these agents. While immunosuppression minimization or withdrawal has been a strategy adopted in response to the development of PTLD and other serious comorbidities, the literature is also revealing for many single-center reports of operationally tolerant patients-defined as those with serially normal liver function tests and serum transaminase levels. Evaluation of allograft dysfunction is complex. Biochemical markers of graft injury have variable sensitivity and specificity, and histological evidence of graft injury may be present even with concurrent normal liver test results. ${ }^{5,6}$ Chronic allograft injury, both inflammation and/or fibrosis, was reported in a multicenter cohort of stable, long-term pediatric LT recipients with consistently normal results from liver tests. ${ }^{7}$ Complications following protocol liver biopsies performed in post-pediatric LT recipients were infrequent and resolved quickly, with biopsy-related cholangitis occurring only in those patients with underlying biliary strictures. ${ }^{38}$ Liver biopsies suffer from variable interpretation and sampling error, while imaging studies are insensitive. ${ }^{38,39}$ Clinical trials are needed to determine predictors of successful immunosuppression withdrawal to allow for personalization of medication regimens.

\section{Recommendations}

- Develop biomarkers that can be used to identify tolerance, graft injury, and injury mechanism(s).

- Assess the effects of immunosuppression personalization on patient outcomes and costs, including targeted therapy to mitigate graft injury before the development of irreversible allograft damage.

\subsection{2 | Metrics beyond the graft}

Measuring outcomes after pediatric LT requires a focus beyond examining only easily accessible data from national administrative or even multicenter clinical databases. An appreciation of the insufficient spotlight to-date on patient centeredness is increasingly being recognized across multiple chronic conditions including adult transplant recipients. ${ }^{40}$ In pediatrics, understanding what is important and most meaningful to our pediatric patients also mandates attention to their parents and siblings, transition planning and community support. This will also impact the quality of life years restored, including the multi-dimensional elements of health status, wellness, vitality, and perceptions of quality of life.

After the first year post-LT, pediatric LT recipients move into a chronic management phase in which the primary goal shifts to sustained graft health without comorbidities or sequelae from long-term immunosuppression. Based on this premise, a composite description of ideal health status in pediatric long-term follow-up patients was derived using the SPLIT database, laying the foundation for future initiatives to focus beyond individual risks of known complications and identify broader sets of outcomes at earlier stages. ${ }^{4}$ An additional emerging theme in health care is recognition of the impact of condition on emotional or functional disabilities as reported by patients. PROs are defined as "any report of the status of a patient's health condition that comes directly from the patient, without interpretation of the patient's response by a clinician or anyone else." ${ }^{141}$ Patient Reported Outcome Measure tools (PROMs) that enable assessment of patient-reported health status for physical, mental, and social well-being have been catalogued within the National Institute of Health's Roadmap Network Project called Patient Reported Outcomes Measurement Information system (PROMIS). ${ }^{42}$ Disease-specific health-related quality of life (HRQOL) tools for pediatric LT are now available and may inform us about challenges from the patients and family perspective that can be leveraged to guide and improve care and facilitate research efforts. ${ }^{43}$ In response to the parents' request for pre-emptive and systematic assessment of the "patient voice" at follow-up ambulatory LT clinic visits by the Patient Advocacy Working Group in the newly formed SNEPT, a learning network that engages families and providers, six pediatric LT programs are currently piloting the feasibility of systemic administration of, the first pediatric liver transplant-specific quality of life tool (Pediatric Liver Transplant Quality of Life, PeLTQL $®)^{43}$ into the clinic environment. ${ }^{44}$

\section{Recommendation}

- Incorporate validated PROMs into the surveillance plans of longterm pediatric LT survivors to amplify the patient voice and focus targets for intervention strategies.

- Leverage currently accumulating registry data and advanced analytics to define composite outcomes and inform targets for improvement within pediatric LT.

\subsection{3 | Non-adherence}

Non-adherence with immunosuppressive medication remains the most frequent cause for late allograft rejection and a leading cause of organ loss and morbidity in children and adolescents who have undergone LT. ${ }^{45}$ Understanding the long-term trajectory of nonadherence to immunosuppression medications can inform decisions regarding liver allocation, adherence monitoring, long-term 
graft health, and targeting intervention efforts. Interventions targeting self-management skills have been effective in improving medication adherence in other pediatric chronic illness groups, with the challenge being time and resource constraints. The demand for the use of technology (such as mobile health and others) to address the barriers associated with non-adherence facilitates the acquisition of the skills needed to independently manage medication regimens. ${ }^{46}$ Despite a growing recognition of this issue, there is currently no internationally accepted or "gold standard" method to assess IS adherence. A recent prospective multicenter study found that MLVI is associated with late acute rejection in pediatric $L T$ recipients. ${ }^{47}$

\section{Recommendations}

- Further, validate and implement broader use of MLVI and other tools to identify at-risk non-adherent patients for interventional strategies.

- Develop collaborative partnerships with healthcare innovators and learning networks to enhance the assessment and delivery of strategies that measure and mitigate non-adherence.

\subsection{4 | Transition and transfer from pediatric to adult transplant care}

The transition from pediatric to adult-centered health care is part of the developmental process for those with chronic childhood diseases and disabilities. ${ }^{46}$ The AST has highlighted transition as an obstacle to longterm graft health for pediatric organ transplant recipients. ${ }^{48}$ Uninformed transition and transfer practices may negatively impact successful selfmanagement, adherence, and-ultimately-graft survival. ${ }^{49-51}$

\section{Recommendations}

- Perform studies that define metrics of a "successful" transition.

- Develop partnerships between pediatric and adult teams to address the domains of self-management and transition readiness.

\section{2 | SUMMARY}

Emerging models in organizational behavior and learning networks provide ample opportunities for our community to explore and invest in, with the goal of decreasing the time from discovery and acquisition of new knowledge to action and impact in pediatric LT. Successful strategies must address problems based on an understanding of the outcomes (metrics) and the gaps between actual and desired outcomes, decision-making (determining how best to address the gap), execution of meaningful research, and policy development or improvement. Figure 2 provides a summary of recommendations and strategies targeting currently identified barriers encountered in the survive and thrive journey traversed by a pediatric LT candidate.

To address the challenges encountered by patients, families, and the healthcare team alike, multi-institutional collaborations have developed strategies to drive awareness, to focus research efforts, and to target quality improvement measures that supplement regulatory efforts in the pediatric LT population. The Studies

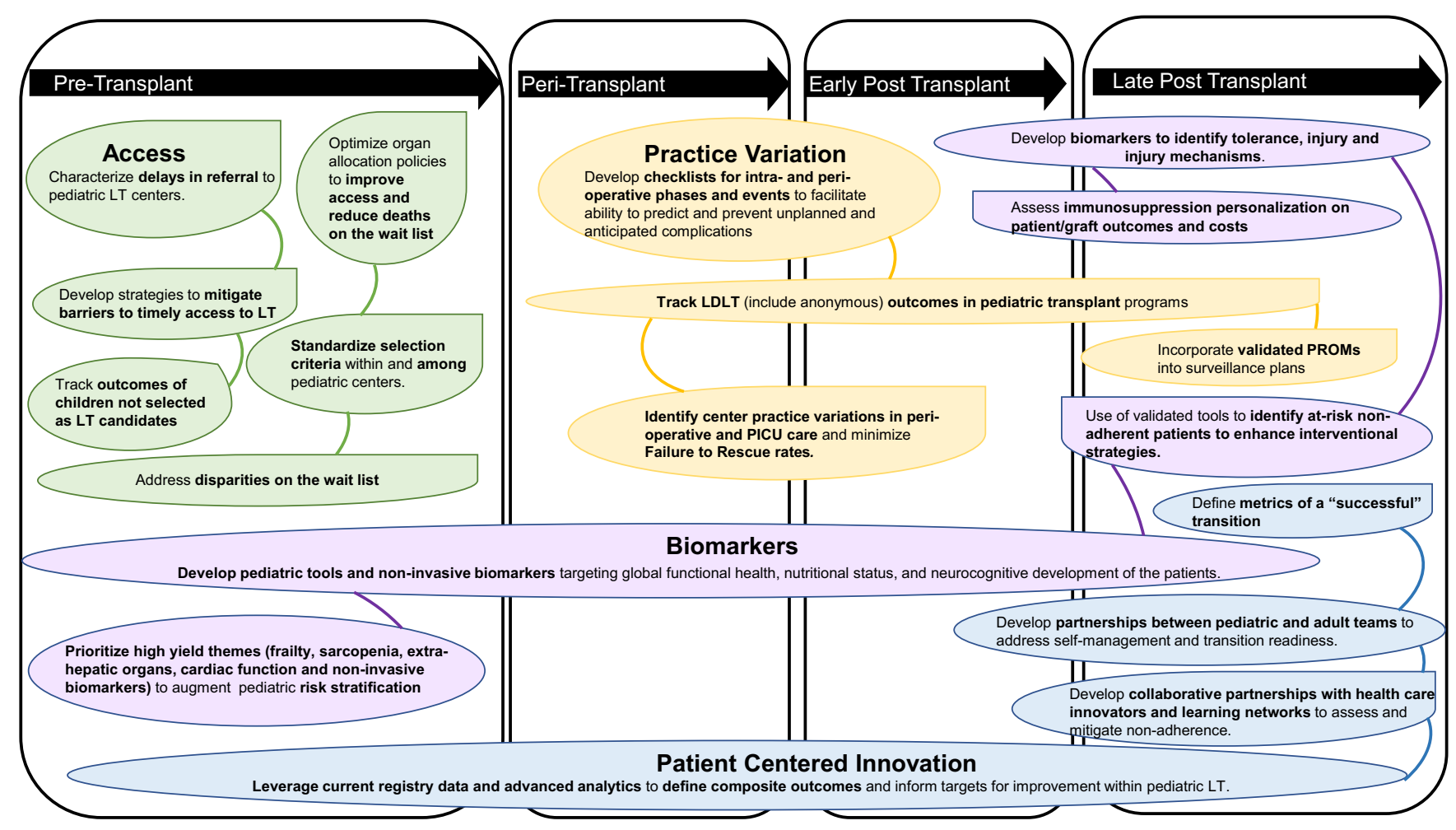

FIGURE 2 Summary of recommendations 
in Pediatric Liver Transplantations consortium founded in 1995 recently incorporated, formally changed its name to become The Society of Pediatric Liver Transplantation (enabling retention of its long-standing acronym-SPLIT), acquired tax-exempt status with a 501c(3), and become the newest section of The Transplantation Society (https://www.tts.org/split/about-us-split). SPLIT remains unwaveringly committed to addressing the many unanswered questions and opportunities illuminated by the barriers articulated in this manuscript. The SPLIT Registry (https://secure.emmes.com/emmesweb) will continue to be the foundational resource to support future initiatives within the SPLIT mission to improve the outcomes in children receiving LT through research, quality improvement, education, advocacy, active engagement of patient and engaged partners/stakeholders, and mentorship of the next generation of clinical care providers and investigators.

The newly formed SNEPT (www.starzltransplantnetwork.com) with its mission "to unite big data, technology, patient advocacy and transplant thought leaders to deliver the best possible care and develop new, scalable solutions to pediatric transplantation's most challenging problems" is a key partner in furthening the SPLIT mission and vision ${ }^{52,53}$ Collaborative efforts within the LICOP, Pediatric Subcommittee, American Society of Transplantation-PCOP, and other stakeholder organizations will be vital in order to move the field forward. We propose an integrated approach of targeted research and policy changes to ensure the best outcomes for this patient population.

\section{ACKNOWLEDGEMENT}

We thank Dr. Jim Squires for his help with Figure 2, and Ms. Anusree Subramonian for her technical support.

\section{AUTHOR CONTRIBUTIONS}

Vicky Lee Ng, George V. Mazariegos, Beau Kelly, Simon Horslen and John C. Bucuvalas: Participated in research design, data acquisition, analysis, and interpretation; drafting and revising the work for important intellectual content; and final approval of the version to be published. Sue V McDiarmid, John C.Magee, Kathleen M. Loomes, Ryan T Fischer, Shikha S. Sundaram, Jennifer C Lai and Helen S. Te: Drafting and revising the work for important intellectual content and final approval of the version to be published.

\section{ORCID}

Vicky Lee Ng (iD https://orcid.org/0000-0002-9998-5692

George V. Mazariegos iD https://orcid.org/0000-0002-2624-8632

Simon Horslen (iD https://orcid.org/0000-0001-5949-7363

\section{REFERENCES}

1. Starzl TE, Koep LJ, Schroter GP, Halgrimson CG, Porter KA, Weil R 3rd. Liver replacement for pediatric patients. Pediatrics. 1979;63(6):825-829.
2. Kehar M, Parekh RS, Stunguris J, et al. Superior outcomes and reduced wait times in pediatric recipients of living donor liver transplantation. Transplant Direct. 2019;5(3):e430-e430.

3. Wadstrom J, Ericzon BG, Halloran PF, et al. Advancing transplantation: new questions, new possibilities in kidney and liver transplantation. Transplantation. 2017;101(Suppl 2S):S1-S41.

4. Ng VL, Alonso EM, Bucuvalas JC, et al. Health status of children alive 10 years after pediatric liver transplantation performed in the US and Canada: report of the studies of pediatric liver transplantation experience. J Pediatr. 2012;160(5):820-826.

5. Ekong UD. The long-term liver graft and protocol biopsy: do we want to look? What will we find? Curr Opin Organ Transplant. 2011;16(5):505-508.

6. Evans HM, Kelly DA, McKiernan PJ, Hubscher S. Progressive histological damage in liver allografts following pediatric liver transplantation. Hepatology. 2006;43(5):1109-1117.

7. Feng S, Bucuvalas JC, Demetris AJ, et al. Evidence of chronic allograft injury in liver biopsies from long-term pediatric recipients of liver transplants. Gastroenterology. 2018;155(6):1838-1851.e1837.

8. Kaplan RM, Frosch DL. Decision making in medicine and health care. Annu Rev Clin Psychol. 2005;1:525-556.

9. Fischler B, Baumann U, D'Agostino D, et al. Similarities and differences in allocation policies for pediatric liver transplantation across the world. J Pediatr Gastroenterol Nutr. 2019.

10. Squires $\mathrm{RH}, \mathrm{Ng} \mathrm{V}$, Romero $\mathrm{R}$, et al. Evaluation of the pediatric patient for liver transplantation: 2014 practice guideline by the American Association for the Study of Liver Diseases, American Society of Transplantation and the North American Society for Pediatric Gastroenterology, Hepatology, and Nutrition. J Pediatr Gastroenterol Nutr. 2014;59(1):112-131.

11. Mathur AK, Ashby VB, Fuller DS, et al. Variation in access to the liver transplant waiting list in the United States. Transplantation. 2014;98(1):94-99.

12. Mazariegos G, Shneider B, Burton B, et al. Liver transplantation for pediatric metabolic disease. Mol Genet Metab. 2014;111(4):418-427.

13. Hansen K, Horslen S. Metabolic liver disease in children. Liver Transpl. 2008;14(5):713-733.

14. Ezekian B, Mulvihill MS, Schroder PM, et al. Improved contemporary outcomes of liver transplantation for pediatric hepatoblastoma and hepatocellular carcinoma. Pediatr Transplant. 2018;22(8):e13305.

15. Sorensen LG, Neighbors K, Martz K, Zelko F, Bucuvalas JC, Alonso EM. Cognitive and academic outcomes after pediatric liver transplantation: functional Outcomes Group (FOG) results. Am J Transplant. 2011;11(2):303-311.

16. Chang C-C, Bryce CL, Shneider BL, et al. Accuracy of the pediatric end-stage liver disease score in estimating pretransplant mortality among pediatric liver transplant candidates. JAMA Pediatr. 2018;172(11):1070-1077.

17. Harris C, Allen K, Waller C, Brooke V. Sustainability in health care by allocating resources effectively (SHARE) 3: examining how resource allocation decisions are made, implemented and evaluated in a local healthcare setting. BMC Health Serv Res. 2017;17(1): 340.

18. Braun HJ, Perito ER, Dodge JL, Rhee S, Roberts JP. Nonstandard exception requests impact outcomes for pediatric liver transplant candidates. Am J Transplant. 2016;16(11):3181-3191.

19. Ge J, Hsu EK, Bucuvalas J, Lai JC. Deceased pediatric donor livers: how current policy drives allocation and transplantation. Hepatology. 2019;69(3):1231-1241.

20. Kim WR, Lake JR, Smith JM, et al. OPTN/SRTR 2013 Annual data report: liver. Am J Transplant. 2015;15(Suppl 2):1-28.

21. Perito ER, Mogul DB, VanDerwerken D, et al. The impact of increased allocation priority for children awaiting liver transplant: a liver simulated allocation model (LSAM) analysis. J Pediatr Gastroenterol Nutr. 2019;68(4):472-479. 
22. Hsu EK, Shaffer M, Bradford M, Mayer-Hamblett N, Horslen S. Heterogeneity and disparities in the use of exception scores in pediatric liver allocation. Am J Transplant. 2015;15(2):436-444.

23. Gorgis NM, Kennedy C, Lam F, et al. Clinical consequences of cardiomyopathy in children with biliary atresia requiring liver transplantation. Hepatology. 2019;69(3):1206-1218.

24. Lai JC, Segev DL, McCulloch CE, Covinsky KE, Dodge JL, Feng S. Physical frailty after liver transplantation. Am J Transplant. 2018;18(8):1986-1994.

25. Lai JC. Editorial: advancing adoption of frailty to improve the care of patients with cirrhosis: time for a consensus on a frailty index. Am J Gastroenterol. 2016;111(12):1776-1777.

26. Lai Jennifer C, Sonnenday Christopher J, Tapper Elliot B, et al. Frailty in liver transplantation: An expert opinion statement from the American Society of Transplantation Liver and Intestinal Community of Practice. American Journal of Transplantation. 2019;19(7): 1896-1906.

27. Lurz E, Quammie C, Englesbe M, et al. Frailty in children with liver disease: a prospective multicenter study. J Pediatr. 2018;194(109-115): e104.

28. Lurz E, Patel H, Frimpong RG, et al. Sarcopenia in children with end-stage liver disease. J Pediatr Gastroenterol Nutr. 2018;66(2): 222-226.

29. Carey EJ, Lai JC, Sonnenday C, et al. A north American expert opinion statement on sarcopenia in liver transplantation. Hepatology. 2019. https://doi.org/10.1002/hep.30828

30. Chaudhary N, Varma V, Kapoor S, Mehta N, Kumaran V, Nundy S. Implementation of a surgical safety checklist and postoperative outcomes: a prospective randomized controlled study. J Gastrointest Surg. 2015;19(5):935-942.

31. Gladwell M. Outliers: The Story of Success. Boston, MA: Little, Brown and. Company Publishing; 2008.

32. Gawande A. Checklist Manifesto: How to Get Things Right. New York, NY: Henry Holt and Company; 2009.

33. Loh C-P, Croome KP, Burcin Taner C, Keaveny AP. Bias-corrected estimates of reduction of post-surgery length of stay and corresponding cost savings through the widespread national implementation of fast-tracking after liver transplantation: a quasi-experimental study. J Med Econ. 2019;1-7.

34. Gurnaney HG, Cook-Sather SD, Shaked A, et al. Extubation in the operating room after pediatric liver transplant: A retrospective cohort study. Paediatr Anaesth. 2018;28(2):174-178.

35. Cramm SL, Waits SA, Englesbe MJ, et al. Failure to rescue as a quality improvement approach in transplantation: a first effort to evaluate this tool in pediatric liver transplantation. Transplantation. 2016;100(4):801-807.

36. Rela M, Reddy MS. "Failure to Rescue" as a novel quality metric in pediatric liver transplantation. Transplantation. 2016;100(4):707.

37. Nazzal M, Lentine KL, Naik AS, et al. Center-driven and clinically driven variation in US liver transplant maintenance immunosuppression therapy: a national practice patterns analysis. Transplant Direct. 2018;4(7):e364.

38. Perito ER, Martinez M, Turmelle YP, et al. Posttransplant biopsy risk for stable long-term pediatric liver transplant recipients: 451 percutaneous biopsies from two multicenter immunosuppression withdrawal trials. Am J Transplant. 2019;19(5): 1545-1551.
39. Rosenberg W, Voelker M, Thiel R, et al. Serum markers detect the presence of liver fibrosis: a cohort study. Gastroenterology. 2004;127(6):1704-1713.

40. Brett KE, Bennett A, Fergusson N, Knoll GA. Quality metrics in solid organ transplantation: protocol for a systematic scoping review. Systematic Rev. 2016;5:99-99.

41. US Department of Health and Human Services FDA Center for Drug Evaluation and Research, US Department of Health and Human Services FDA Center for Biologics Evaluation and Research, US Department of Health and Human Services FDA Center for Devices and Radiological Health. Guidance for industry: patient-reported outcome measures: use in medical product development to support labeling claims: draft guidance. Health Qual Life Outcomes. 2006;4(1):79.

42. Fries JF, Bruce B, Cella D. The promise of PROMIS: using item response theory to improve assessment of patient-reported outcomes. Clin Exp Rheumatol. 2005;23(5 Suppl 39):S53-57.

43. Ng V, Nicholas D, Dhawan A, et al. Development and validation of the pediatric liver transplantation quality of life: a disease-specific quality of life measure for pediatric liver transplant recipients. J Pediatr. 2014;165(3):547-555.

44. Squires JE. LB, Lorts A.,. A Learning Health Network for Pediatric Liver Transplantation: Inaugural Meeting Report from the Starzl Network for Excellence in Pediatric Transplantation. Pediatr Transplantation. 2019; (Accepted for publication).

45. Shemesh E, Shneider BL, Savitzky JK, et al. Medication adherence in pediatric and adolescent liver transplant recipients. Pediatrics. 2004;113(4):825-832.

46. Fredericks EM. Transition readiness assessment: The importance of the adolescent perspective. Pediatr Transplant. 2017;21(3):e12921.

47. Shemesh E, Bucuvalas JC, Anand R, et al. The Medication Level Variability Index (MLVI) predicts poor liver transplant outcomes: a prospective multi-site study. Am J Transplant. 2017;17(10):2668-2678.

48. American Society of Transplantation. Pediatric transition portal. https://www.myast.org/education/specialty-resources/peds-trans ition; 2018. Accessed 25 October, 2018.

49. Watson AR. Non-compliance and transfer from paediatric to adult transplant unit. Pediatr Nephrol. 2000;14(6):469-472.

50. Annunziato RA, Bucuvalas JC, Yin W, et al. Self-management measurement and prediction of clinical outcomes in pediatric transplant. J Pediatr. 2018;193(128-133):e122.

51. Annunziato RA, Freiberger D, Martin K, Helcer J, Fitzgerald C, Lefkowitz DS. An empirically based practice perspective on the transition to adulthood for solid organ transplant recipients. Pediatr Transplant. 2014;18(8):794-802.

52. Billett AL, Colletti RB, Mandel KE, et al. Exemplar pediatric collaborative improvement networks: achieving results. Pediatrics. 2013;131(Suppl 4):S196-203.

53. Clancy CM, Margolis PA, Miller M. Collaborative networks for both improvement and research. Pediatrics. 2013;131(Suppl 4):S210-214.

How to cite this article: Ng VL, Mazariegos GV, Kelly B, et al. Barriers to ideal outcomes after pediatric liver transplantation. Pediatr Transplant. 2019;23:e13537. https://doi.org/10.1111/ petr.13537 